\title{
EXPLORACIÓN DEL APRENDIZAJE DE LOS ESTUDIANTES HACIENDO USO DE AMBIENTES COLABORATIVOS: ENSEÑANDO INTELIGENCIA ARTIFICIAL
}

\author{
(EXPLORING THE STUDENT'S LEARNING USING COLLABORATIVE ENVIRONMENTS: \\ TEACHING ARTIFICIAL INTELLIGENCE)
}

Gustavo de la Cruz Martínez, Fernando Gamboa Rodríguez

Universidad Nacional Autónoma (México)

\section{RESUMEN}

Se ha encontrado en los sistemas de cómputo una herramienta útil para apoyar el proceso de enseñanza-aprendizaje. Haciendo uso de las posibilidades que la computadora provee se presentan conceptos e ideas a los estudiantes de manera multimedia, ágil e interactiva, además de que se diseñan ambientes virtuales que les permiten ejercitar y extender sus conocimientos.

A la par de la problemática de diseñar y presentar contenidos didácticos, existen esfuerzos importantes por tratar de identificar y evaluar si el estudiante está comprendiendo de manera adecuada los temas.

En este trabajo se presenta un estudio del aprendizaje de los estudiantes haciendo uso de un ambiente virtual, e-Vitro, que es de apoyo tanto para profesores como para los estudiantes, en un curso de Inteligencia Artificial. El análisis del aprendizaje del estudiante se basa en el modelado de la interacción del estudiante con el software y el análisis automático de agentes de software que él desarrolla para interactuar en el ambiente de e-Vitro.

\begin{abstract}
Computer systems have been widely used as a support tool in the teaching-learning process. The many possibilities that computers offer to teachers make possible for them to create new multimedia, interactive and much more interesting materials that should help students to visualize and grab complex concepts. In the same way, virtual spaces where students can exercise their knowledge and create new one are nowadays available.
\end{abstract}


Gustavo De la CRuz Martínez y Fernando Gamboa

EXPLORACIÓN DEL APRENDIZAJE DE LOS ESTUDIANTES HACIENDO USO DE AMBIENTES COLABORATIVOS: ENSEÑANDO INTELIGENCIA ARTIFICIAL

Besides the problem of presenting information and virtual spaces, is the problem of evaluating the pupils: how much do they understand the concepts reviewed in class? A lot of work is being carried out on this issue too.

In this work we present a study on how to evaluate the correct learning of the students that are using a virtual environment called " $e$-Vitro". $e$-Vitro is a tool that is used by students and teachers in a course of Artificial Intelligence. The analysis of students learning is based on their interaction with the system, as well as on an automatic evaluation performed on the agents they must develop in the $e$-Vitro platform.

\section{INTRODUCCIÓN}

Cuando un profesor presenta el contenido de un curso a sus estudiantes, hace uso de todos los medios a su alcance para lograr que sus pupilos comprendan los conceptos o principios que él indica. En la mayoría de las ocasiones, el mayor reto no radica en el dominio de los contenidos del curso sino en la forma de presentarlos al alumnado. De aquí que, aunque el profesor sea un erudito en el tema, sino es capaz de presentarlo de manera adecuada para que los estudiantes lo comprendan, el resultado será que los estudiantes obtendrán conocimientos mínimos o nulos acerca del tema en cuestión.

En los últimos años se ha encontrado en los sistemas de cómputo una herramienta útil para dos tareas fundamentales en el proceso de enseñanza-aprendizaje: 1) como apoyo en la presentación de contenidos o conceptos complejos a los estudiantes. En este caso, los docentes utilizan los diferentes medios que la computadora provee para presentar a sus estudiantes conceptos e ideas complejas de una manera más clara, esperando que así puedan asimilar más fácilmente los contenidos. 2) como herramienta con la que el estudiante crea su propio conocimiento. Bajo esta dinámica, la computadora se convierte más en un laboratorio y herramienta de exploración que permite a los alumnos, al estudiar y analizar un fenómeno de cualquier índole, al recabar y sintetizar información, o al interpretar resultados, crear su propio conocimiento.

Así, con los recursos que la computadora ofrece, los profesores han desarrollado herramientas que permiten al estudiante no sólo consultar el contenido temático, sino interactuar con ambientes que ponen a prueba sus conocimientos y llevar a la práctica aquellos lineamientos que marca la teoría.

Sin embargo, el diseño y presentación de los contenidos es sólo el principio del proceso enseñanza-aprendizaje, otro elemento importante está vinculado con 
Gustavo De la Cruz Martínez y Fernando Gamboa

EXPLORACIÓN DEL APRENDIZAJE DE LOS ESTUDIANTES HACIENDO USO DE AMBIENTES COLABORATIVOS: ENSEÑANDO INTELIGENCIA ARTIFICIAL

el seguimiento del avance de los estudiantes. Cada estudiante tiene diferentes capacidades y habilidades que le permiten asimilar los conocimientos de diferente manera, por tanto el profesor se enfrenta a la problemática de evaluar el nivel de comprensión del tema de sus estudiantes, para detectar algunas fallas y evitar que dichos estudiantes tengan nociones erróneas al respecto. Nuevamente el profesor echa mano de todos los medios disponibles para detectar si el estudiante está comprendiendo el tema o no; pero es imposible contar con un oráculo que indique al final de cada lección o tema el nivel de comprensión de cada alumno.

Con el uso de sistemas de software es posible poner a prueba los conocimientos del estudiante y así, presentar al profesor resultados que le ayuden a detectar errores de comprensión con respecto a los contenidos que él presenta a sus estudiantes.

En este trabajo se describe un estudio acerca de la enseñanza de la Inteligencia Artificial a nivel de licenciatura, que utiliza un ambiente colaborativo, e-Vitro, como una herramienta de apoyo para el estudiante. A partir del uso de este software el profesor cuenta con información que le ayuda a determinar si el estudiante ha comprendido los principios básicos que se le han presentado y si es capaz de poner en práctica tales conceptos.

Este trabajo está organizado de la siguiente manera, en la primera parte se describen las problemas más comunes relacionados con la enseñanza de la Inteligencia Artificial de donde se observa la importancia de tener herramientas de seguimiento del aprendizaje del estudiante, a continuación se describen los aspectos importantes de e-Vitro, una herramienta que permiten realizar un análisis acerca de los conocimiento del usuario. Finalmente, se presentan las conclusiones generales de este proyecto.

\section{ENSEÑANDO INTELIGENCIA ARTIFICIAL}

\section{Evaluación y seguimiento del aprendizaje de los estudiantes}

Aún con el uso de herramientas que refuercen el aprendizaje no se puede saber nada acerca del conocimiento del estudiante, hasta no poner a prueba dichos conocimientos. Tradicionalmente se han diseñado elementos de evaluación para conocer esta información, pero existen muchas áreas del conocimiento donde es muy difícil diseñar evaluaciones que brinden suficiente información al profesor sobre sus estudiantes. 
Gustavo De la CRuz Martínez y Fernando Gamboa

EXPLORACIÓN DEL APRENDIZAJE DE LOS ESTUDIANTES HACIENDO USO DE AMBIENTES COLABORATIVOS: ENSEÑANDO INTELIGENCIA ARTIFICIAL

El caso de la inteligencia Artificial (IA) es un ejemplo claro de la dificultad de evaluar a los estudiantes cuando se les enseñan las nociones básicas, como es la solución de problemas usando heurísticas. No basta sólo con determinar si el estudiante conoce las cualidades generales de las diferentes técnicas heurísticas, sino que él debe estar conciente de las limitaciones de cada una de ellas y de los problemas relacionados con su implementación.

En muchos casos, el profesor evalúa los detalles de estas implementaciones mediante la asignación de prácticas donde se presentan problemas que el estudiante debe resolver con alguna o algunas de las técnicas estudiadas. Para determinar si la solución propuesta por los estudiantes es adecuada se debe analizar a detalle la implementación, lo cual no siempre es una tarea fácil.

Este tipo de evaluaciones presenta ciertas limitaciones; por un lado, se puede evaluar si la implementación del estudiante es capaz de resolver el problema en cuestión, pero puede resultar difícil analizar todas las cualidades de la solución propuesta, ya sea por falta de tiempo o por la dificultad de modificar e interactuar con la solución del estudiante.

Por otro lado, el profesor debe analizar el trabajo final del estudiante, pero todos los puntos intermedios para llegar a la solución final no pueden ser observados, y de estos primeros intentos se podría obtener mucha información acerca de lo que el estudiante está entendiendo acerca del tema en cuestión.

Bajo este panorama es importante que un profesor cuente con mejores medios que le permitan identificar problemas en el aprendizaje de sus estudiantes.

\section{Uso de ambientes colaborativos: e-Vitro}

Cuando se inicia la enseñanza de la IA, la transmisión de los fundamentos e ideas principales de esta disciplina puede dificultarse por la necesidad de aplicar los conceptos a la resolución de problemas, con la inconveniente consecuencia de que los estudiantes suelen preocuparse más por los detalles relacionados con la implementación de los conceptos que por el análisis de cualidades y limitaciones de dichos conceptos. Por tal razón, es necesario contar con herramientas que permitan al estudiante y al profesor concentrarse en los puntos esenciales y, por otra parte, poder calificar el avance de los estudiantes. Un ejemplo de las herramientas desarrolladas con este fin es e-Vitro (De la Cruz \& Juárez, 2005), su objetivo es proveer un mundo virtual donde los estudiantes ponen en práctica los conocimientos teóricos vistos 
Gustavo De la Cruz Martínez y Fernando Gamboa

EXPLORACIÓN DEL APRENDIZAJE DE LOS ESTUDIANTES HACIENDO USO DE AMBIENTES COLABORATIVOS: ENSEÑANDO INTELIGENCIA ARTIFICIAL

en un curso básico de IA, haciendo uso de una biblioteca de programación que contiene las implementaciones de los algoritmos elementales para la resolución de problemas.

e-Vitro es una herramienta que permite al usuario construir seres virtuales o agentes, queinteractúan de manera independiente entre sí en un ambiente controlado, que va presentando diferentes dificultades a cada uno de estos seres. Cada estudiante define el comportamiento de sus agentes haciendo uso de los conceptos básicos de la Inteligencia Artificial, poniendo a prueba las diferentes técnicas en este tipo de entornos y de esta manera saber las ventajas y limitaciones de cada una de ellas. Los mecanismos de comportamiento diseñados por el estudiante van a la par de los conocimientos que va recibiendo en la clase de IA, de tal suerte que, pueda analizar, discutir y desarrollar ideas para que su agente pueda sobrevivir en el mundo de eVitro.

El problema que plantea el mundo de e-Vitro es el siguiente:

Hay una persona perdida en un valle. En éste lugar como en todo valle se tienen obstáculos: un río, formaciones rocosas, montañas, bosques, animales peligrosos, etc. (figura 1).

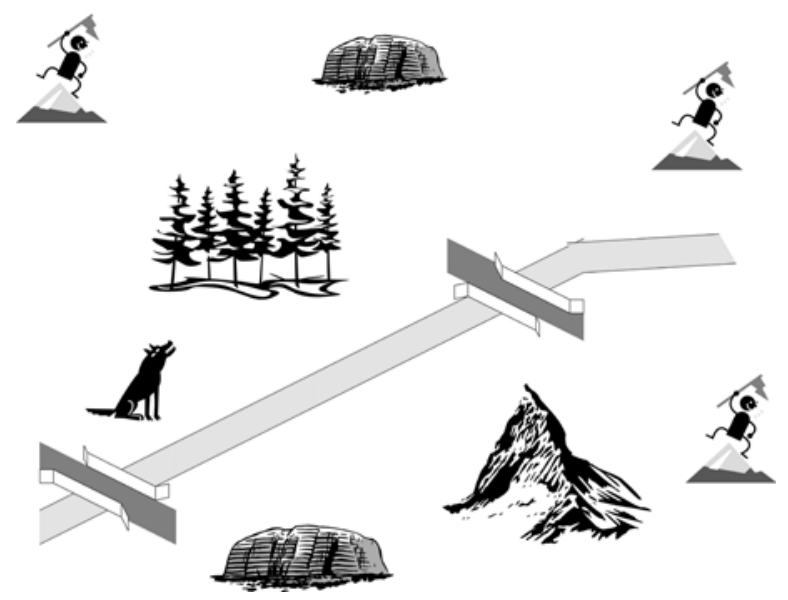

Fig. 1: Mapa simbólico del mundo de e-Vitro

Haciendo una abstracción y simplificación de dicho problema, el mundo de eVitro es observado como (figura 2): 
Gustavo De la Cruz Martínez y Fernando Gamboa

EXPLORACIÓN DEL APRENDIZAJE DE LOS ESTUDIANTES HACIENDO USO DE AMBIENTES COLABORATIVOS: ENSEÑANDO INTELIGENCIA ARTIFICIAL

- Un espacio cuadriculado, para facilitar el concepto de desplazamiento.

- Varias zonas denominadas tierras altas, las cuales siempre representarán el objetivo final al que debe llegar el agente del alumno (en gris).

- Existen áreas reservadas que representan obstáculos que impiden el desplazamiento (en negro).

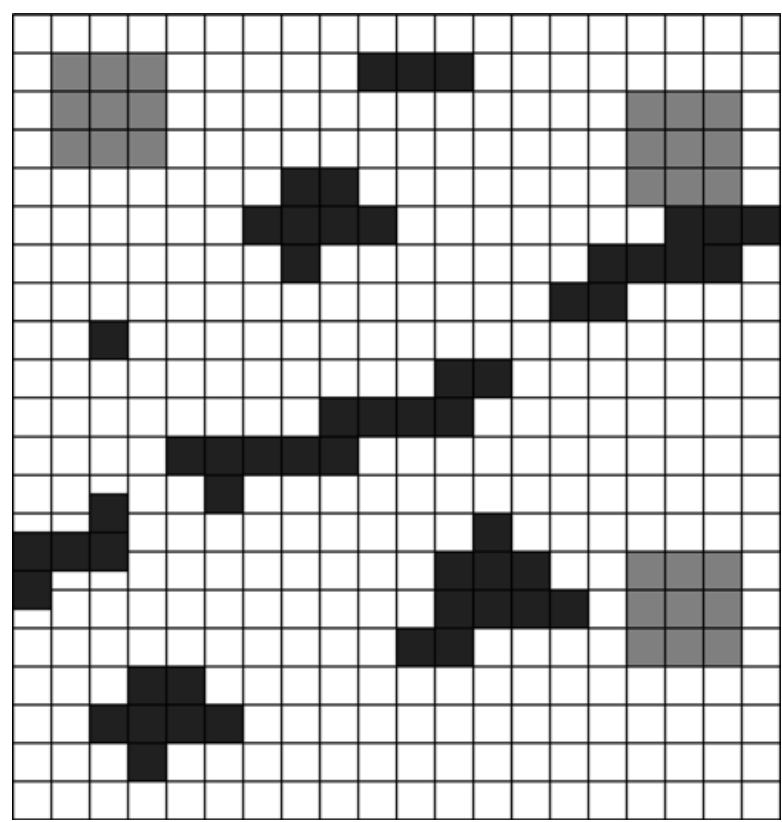

Fig. 2: Esquema de e-Vitro

\section{EXPLORACIÓN DEL APRENDIZAJE DE LOS ESTUDIANTES}

\section{¿Cómo analizar el aprendizaje de los estudiantes?}

Bajo las situaciones anteriores es inevitable cuestionarse acerca de la capacidad de las herramientas basadas en sistemas de cómputo para brindarnos información acerca del aprendizaje del estudiante. Este cuestionamiento puede estudiarse a dos niveles, por un lado, analizar las cualidades pedagógicas de la herramienta y por el 
Gustavo De la Cruz Martínez y Fernando Gamboa

EXPLORACIÓN DEL APRENDIZAJE DE LOS ESTUDIANTES HACIENDO USO DE AMBIENTES COLABORATIVOS: ENSEÑANDO INTELIGENCIA ARTIFICIAL

otro los aspectos relacionados con el uso de la herramienta por parte del estudiante. Este último aspecto es el que nos ocupa, es decir, cómo puedo analizar el conocimiento de un estudiante a partir del uso de una herramienta de software.

En un ambiente como el de e-Vitro, podemos estudiar el proceso de enseñanzaaprendizaje, por un lado tenemos la teoría sobre representación del conocimiento y solución de problemas haciendo uso de las búsquedas y, por el otro, las ideas y conceptos que el estudiante se forma sobre estos mecanismos y que se implementó para la definición del comportamiento de sus agentes. La cuestión que nos interesa resolver es: ¿cómo es el concepto que el usuario tiene de las diferentes técnicas usadas para la resolución de problemas?, y si dicho concepto, es correcto o no. En este trabajo se proponen lineamientos que ayudan al profesor a inferir las características de la solución propuesta por el estudiante, y se provee de una herramienta que le ayude a determinar si sus estudiantes han comprendido los conceptos que se les presentaron en el curso.

La Ingeniería de Sistemas Cognitivos puede ser utilizada para atacar esta cuestión, con el apoyo de la Interacción Humano-Computadora y la Psicología Cognitiva, en particular el área de la Usabilidad, busca entender más acerca del procesamiento de la información y del modelo conceptual que los estudiantes construyen.

En este sentido, un correcto análisis de cómo es el modelo mental que el estudiante asocia al sistema informático requiere que tengamos algunas consideraciones acerca de cómo el humano realiza el procesamiento de la información, con el fin de poder determinar cómo construye los conceptos y cómo interactúa con un sistema. Se han realizado diversas investigaciones entorno a qué es lo que el usuario entiende sobre una aplicación de software, es decir, se ha tratado de buscar mecanismos para determinar qué es el sistema para el usuario. Se cree que una forma de realizar esto es a partir de cómo el usuario usa el sistema. Este punto de vista se basa en la idea de que el sistema fue diseñado para apoyar a una serie de procesos, los cuales fueron esquematizados durante la fase de desarrollo para poder ser presentados al usuario a través de la interfaz y es a partir de ésta última que el usuario construye su propia abstracción del proceso.

Sin embargo, no puede olvidarse que cada persona puede realizar una abstracción diferente de cada proceso y por tanto de todo el sistema. Considerando al ser humano como procesador de información, partimos de que los sentidos proporcionan una imagen del mundo físico. Para extraer el sentido psíquico de la información sensorial es necesaria una interpretación de ella. Para la interpretación se considera que las 
Gustavo De la CRuz Martínez y Fernando Gamboa

EXPLORACIÓN DEL APRENDIZAJE DE LOS ESTUDIANTES HACIENDO USO DE AMBIENTES COLABORATIVOS: ENSEÑANDO INTELIGENCIA ARTIFICIAL

señales son procesadas de acuerdo a la experiencia previa, de tal forma que la memoria se convierte en un factor importante ya que la recuperación de la información pasada influirá notablemente en la comprensión del presente, para finalmente tomar las decisiones que se consideren adecuadas al contexto (Norman, 1991).

\section{Modelado de los estudiantes}

Siguiendo la hipótesis de la comprensión del sistema a partir de su uso, se han realizado diferentes propuestas para modelar al usuario (Girardi, et. al., 2005), por ejemplo, analizando la forma en que las personas usan los sistemas Web se pueden encontrar patrones de uso y con esto determinar modelos implícitos de los posibles usuarios, para finalmente obtener sistemas que se adapten (de forma autónoma o mediante procesos de rediseño) a las necesidades de los usuarios.

Los modelos de los usuarios representan características relevantes de los usuarios que son usadas por los sistemas adaptables para satisfacer, de manera personalizada, las necesidades de una gran diversidad de usuarios. La tecnología de agentes ha sido usada con efectividad en el desarrollo de sistemas adaptables por sus características de autonomía, sociabilidad y aprendizaje.

Los sistemas adaptables se basan en la creencia de que es posible adaptar sistemas de acuerdo al conocimiento y experiencia de los usuarios, las acciones previas, las propiedades cognitivas, objetivos e intenciones del usuario, sus intereses y preferencias. Por lo tanto, los retos para este tipo de tecnologías radican en la determinación de estas cualidades. Pero cuando se pretende estudiar la mecánica del pensamiento humano es necesario considerar diversos puntos de vista, más aún si se pretende llegar a un modelo que será implementado en un programa en computadora.

\section{Exploración del aprendizaje de los estudiantes}

Con el apoyo de e-Vitro se realizan dos tipos de análisis para obtener información acerca del aprendizaje de los estudiantes. El primero es un análisis automatizado de los agentes que el estudiante desarrolla y el segundo, se basa en el seguimiento de cómo el estudiante interactúa con e-Vitro.

El sistema proporciona los resultados de estos dos análisis al profesor quien debe revisarlos y determinar si necesita hacer alguna observación al estudiante. 
Gustavo De la Cruz Martínez y Fernando Gamboa

EXPLORACIÓN DEL APRENDIZAJE DE LOS ESTUDIANTES HACIENDO USO DE AMBIENTES COLABORATIVOS: ENSEÑANDO INTELIGENCIA ARTIFICIAL

\section{Análisis automatizado}

Una vez que cada estudiante desarrolla un agente y lo incorpora al ambiente virtual de e- Vitro, el sistema lo pone a prueba para tratar de determinar cómo es su comportamiento y cuáles son las características de su implementación.

Este análisis supone que el estudiante se basa en el contenido del curso para definir el comportamiento de sus agentes, de manera que el agente debe, por lo menos de manera general, presentar las cualidades y limitaciones de los métodos que ahí se presentan, es decir, si el tema que se está trabajando es la solución de problemas mediante el uso del algoritmo de búsqueda a profundidad (Rich \& Knight, 1991) y se sabe que dicho algoritmo presenta una serie de ventajas y limitaciones, se pondrá a prueba al agente del estudiante para determinar si presenta dichas ventajas y limitaciones. Para realizar esto, el sistema es capaz de crear situaciones en el ambiente virtual que simulen ciertas condiciones que obligan al agente a responder de cierta manera y así saber si presenta o no las características que se esperan.

Para cada técnica se determinaron una serie de cualidades que debe presentar (Rich \& Knight, 1991; Russell \& Norvin, 1995) y el sistema pone a prueba al agente en situaciones diversas para determinar si la implementación logra satisfacer los requerimientos.

\section{Seguimiento de la interacción del estudiante}

Cuando un estudiante se encuentra desarrollando su agente, en la mayoría de los casos no obtiene una implementación adecuada desde su primer intento; sino que, va corrigiendo el comportamiento de su agente hasta obtener uno con el comportamiento que él considere adecuado. Estos primeros intentos y correcciones brindan información que el profesor puede utilizar para determinar si el estudiante está entendiendo el tema en cuestión.

El sistema guarda una bitácora de los intentos que el estudiante realiza, estas bitácoras incluyen el comportamiento paso a paso del agente del estudiante, desde que fue incorporado al ambiente virtual hasta el punto donde deja de existir, o estado actual si aún existe. Otro elemento importante de la bitácora es la estrategia usada por el sistema para poner a prueba al agente.

A partir de esta bitácora, el profesor puede analizar el desempeño del estudiante y detectar problemas con la implementación de los diferentes métodos. 
Gustavo De la CRuz Martínez y Fernando Gamboa

EXPLORACIÓN DEL APRENDIZAJE DE LOS ESTUDIANTES HACIENDO USO DE AMBIENTES COLABORATIVOS: ENSEÑANDO INTELIGENCIA ARTIFICIAL

\section{CONCLUSIONES}

Se ha observado que el uso de herramientas que permitan poner en práctica los conceptos teóricos ayuda al estudiante a entenderlos de manera más adecuada, ya que le sirve para percibir las ventajas y limitaciones de los diferentes conceptos que se le presentan. Esto es especialmente importante en cursos de introducción a un área, ya que en muchas ocasiones dichos cursos duran poco tiempo en comparación con todos los temas que abarcan. En particular con la enseñanza de la Inteligencia Artificial es primordial atraer al estudiante y aclarar la concepción que él tenga del área.

Un aspecto importante del proceso de enseñanza-aprendizaje es el seguimiento del estudiante. Haciendo un análisis de la manera en que los estudiantes usan los sistemas de software que apoyan a los cursos, se puede extraer mucha información acerca de lo que ellos están entendiendo de dicho curso. Un análisis de este tipo se realizó con la herramienta e-Vitro.

En el caso de ambientes colaborativos y autónomos, como e-Vitro, se facilita la recolección de información relacionada con la manera en que el estudiante usa los conceptos que le son presentados en la clase, ya que cada estudiante puede interactuar con el software en el momento que lo desee y el software puede examinar de manera detallada las soluciones propuestas por el estudiante. Pero un punto clave para el correcto funcionamiento de este tipo de herramientas, es el modelo que se use para representar al estudiante y su conocimiento. Por tal motivo, la investigación relacionada con los modelos para representar al usuario de los sistemas de software es útil, tanto para el área de las Ciencias de la Computación, como para todas aquellas que hacen uso del software y les interesa conocer que tan adecuado es para la tarea que apoya.

El modelo utilizado para este trabajo funciona de manera adecuada sólo para el análisis de implementaciones sencillas del estudiante, ya que para implementaciones muy sofisticadas al sistema le es muy difícil determinar las características de la implementación.

Con este tipo de investigaciones se pretende conocer más sobre los mecanismos asociados con los procesos pensamiento, para poder entender mejor el actuar del hombre, en particular, al momento de usar un sistema de cómputo. El conocimiento acerca de este tópico nos permitiría proporcionar mejores medios de comunicación entre el hombre y la computadora. 
Gustavo De la Cruz Martínez y Fernando Gamboa

EXPLORACIÓN DEL APRENDIZAJE DE LOS ESTUDIANTES HACIENDO USO DE AMBIENTES COLABORATIVOS: ENSEÑANDO INTELIGENCIA ARTIFICIAL

\section{REFERENCIAS BIBLIOGRÁFICAS}

Balby, L.; Girardi, R.; de Oliveira, I.; Lindoso, A. (2004). A Development Experience on Agent-based User Modeling. En Proceedings of the Workshop on Architectures and Methodologies for Building AgentBased Learning Environments. Held in Conjunction with SBIA, SBRN, IEEE $M L S P$, Säo Luis, Maranhäo, Brasil: Ed. Livro Rápido, 17-28.

Brusilovsky, P.; Maybury, M. (2002). From Adaptive Hypermedia to the Adaptive Web. Communications of ACM, 45 (5), 31-33.

Bylund, M.; Waern, A. (1997). Adaptation Agents: Proving Uniform Adaptions in Open Service Architectures. En Proceedings Conference on Conceptual Modeling (ER2004), Shangai, China.

De la Cruz, G.; Juárez F., (2005) e-Vitro: un ambiente virtual de apoyo a la enseñanza de la Inteligencia Artificial. En Congreso de Instrumentación SOMI XX, SOMI (Eds.), León, México.

Fink, J., Kobsa, A. (2002). User Modeling in Personalized City Tours. Artificial Intelligence Review, 18 (1), 33-74.

Kobsa, A. (1994). User Modeling and UserAdapted Interaction. En Conference Companion on Human factors in computing systems, Boston, Mass, United States, 415-416.

Kobsa, A.; Koenemann, J., Pohl, W. (1999). Personalized hypermedia Presentation Techniques for Improving Online Customer Relationships. GMD Report 66.

Girardi, R. (2002). Reuse in Agent-based Application Development. En First International Workshop on Software Engineering for Large-Scale MultiAgent Systems (SELMAS'2002).
Girardi, R.; Balby, L.; Ribeiro, I. (2005). A system of agent-based software patterns for user modeling based on usage mining. Interacting with computers, 17, 567-591.

Girardi, R.; Faria, C. (2003). A Generis Ontology for the Specification of Domain Models. In: Overhage, Sven, Turowski, Klaus (Eds.), En Proceedings of First International Workshop on Component Engineering Methodology (WCEM'03) at Second International Conferenceon Generative Programming and Component Engineering, Erfurt, Germany, 41-50.

Langley, P. (1999). User Modeling in Adaptive Interfaces. En Proceeding of the Seventh International Conference on User Modeling, Springer. Banff, Canada, 357-370.

Nick, Z.; Themis, P. (2001). Web search using a genetic algorithm. IEEE Internet Computing, 5 (2), 18-26.

Matz, M. (1980). Towards a Computacional Theory of Algebraic Competente. The Journal of Mathematical Behavior, 3 (1), 93-166.

Norman, D. A. (1991). El Procesamiento de la imagen en el hombre, memoria $y$ atención. Buenos Aires: Paidos Studio.

Pierrakos, D.; Paliouras, G.; Papatheodorou, C.; Spyropolous, C.D.(2003). Webusage mining as a tool for personalization: a survey. User Modeling and UserAdapted Interaction, 13, 311-372.

Russell, S.; Norving, P. (1995). Artificial Intelligence: A Modern Approach. Prentice Hall, Englewood Cliffs, NJ. 
Gustavo De la Cruz Martínez y Fernando Gamboa

EXPLORACIÓN DEL APRENDIZAJE DE LOS ESTUDIANTES HACIENDO USO DE AMBIENTES COLABORATIVOS: ENSEÑANDO INTELIGENCIA ARTIFICIAL

\section{PALABRAS CLAVE}

Ambientes colaborativos, técnicas de modelado de usuario.

\section{KEYWORDS}

Collaborative environments, user modeling techniques.

\section{PERFIL ACADÉMICO DE LOS AUTORES}

De la Cruz Martínez Gustavo < gustavo.delacruz@ccadet.unam.mx> Línea de investigación: Sistemas e Interfaces adaptables.

Dr. Gamboa Rodríguez Fernando <fernando.gamboa@ccadet.unam.mx> Línea de investigación: Interacción Humano Computadora, Diseño de Software Educativo Centrado en el Estudiante.

Dirección postal:

Laboratorio de Interacción Humano Máquina y Multimedios, Centro de Ciencias Aplicadas y Desarrollo Tecnológico Universidad Nacional Autónoma de México

Circuito Exterior S/N, Ciudad Universitaria, 04510 México, D.F.

Fecha de entrega: 23.10.06

Fecha de aceptación: 20.12.06 